\title{
Reconstruction of the image objects by SAIF
}

\author{
Subrahmanyam.Ch ${ }^{1}$, Ch.Janaki Devi ${ }^{2}$, Dr.V.Sailaja ${ }^{3}$, K.Jyothi ${ }^{4}$ \\ ${ }^{I}$ Department of Technical Education Andhra Pradesh, Hyderabad \\ ${ }^{2,3,4}$ Godavari Institute of Engineering \& Technology
}

\begin{abstract}
In this paper, I arranged the damaged or different pieces of the image in proper manner using Special Adjacent Image Fragments (SAIF). An integrated method for automatic color based 2-D image fragment reassembly is presented. The proposed 2-D reassembly technique is divided into four steps. Initially, the image fragments which are probably spatially adjacent are identified utilizing techniques employed in content based image retrieval systems. The second operation is to identify the matching contour segments for every retained couple of image fragments, via a dynamic programming technique. We have experimented with the proposed method using digitally scanned images of actual torn pieces of paper image prints and we produced very satisfactory reassembly results.
\end{abstract}

\section{Introduction}

In our work, the automated reassembly of images from fragments follows a four step model, similar to the one presented for 3-D object reconstruction. The first step of our approach is the identification of probable adjacent image fragments, in order to reduce the computational burden of the subsequent steps. There, several color-based techniques are employed.

However, as stated in a following section, this step can be omitted sacrificing the lower running time of the image reassembly procedure, for higher performance. Once the matching contour segments are identified, a third operation takes place. Here, the geometrical transformation, which best aligns two fragment contours along their matching segments, is found. Several such approaches exist, however, most of them are either not robust to matching errors and/or have high computational complexity. Our goal is to investigate and propose the most robust techniques in order to produce accurate results at each intermediate step. The alignment transformation of the fragments is simultaneously found during matching.

After the discovery of matching contour segments, the final reassembly step performs two actions, namely matching relaxation and fragments merging. During matching relaxation, every pair of aligned input fragments is checked for overlap along their matching contour segments. If they overlap, this matching is discarded.

Otherwise, the neighboring fragments of this pair are identified. A score, called support, is assigned to the neighborhood of each pair of non-overlapping fragments. This score increases as the number of neighboring fragments as well as the matching confidence assigned to pairs of fragments increase. It is clear that the problem of 2-D puzzle assembly does not meet the major difficulty of the image and object reconstruction problems; that is the missing or highly damaged image (or) object fragments. Thus, in general, the algorithms proposed in this field would be inadequate to solve such problems.

\section{FRAGMENTS}

\section{Spatial Adjacent Image}

Color quantization can be based on a commercial color palette, e.g., the Gretag Macbeth Color Checker. The Macbeth Palette can be used to evaluate color reproduction systems and it consists of 24 different colors that are scientifically chosen to represent a variety of naturally occurring colors. Color quantization is used to find the normalized quantized color image histograms, which can be used for color image retrieval.

We have also experimented with the Spatial Chromatic Histogram, which provides information both of color presence and color spatial distribution. Let us suppose that we have a set of image fragments where we have to find their matching contour segments. This set may contain the image fragment pairs identified by the previous step or all the image fragment pairs (if the first step has been skipped). This operation is performed on image fragment pairs.

In this section, we present a novel algorithm for identifying matching contour segments of couples of input fragments. Our approach to fragment contour matching is based exclusively on information regarding the color of their contours. We investigate methods for finding the best geometrical transformation that aligns fragment contours along their matching segments. 
All matching contour segments identified during the previous steps must be properly aligned, before the reassembly of the overall image. The disadvantages of the alignment algorithms found in the literature have led us to use variants of the ICP algorithm for contour alignment. The original form of the ICP algorithm is not robust to outliers, since it does not trim noisy data. Such outliers, coming from errors during the contour segment matching, create serious problems to alignment, if not properly handled. In order to obtain ICP versions that are robust to outliers, many ICP variants have been proposed in the literature. These variants have been classified into six categories. In order to develop a robust contour alignment procedure, we have studied three popular robust versions of ICP and have evaluated their performance.

\section{REASSEMBLY STEPS}

\section{Experiment}

Whether executing (or not) the first step of the proposed method, the contour segment matching for every input couple of image fragments must be done. When the first step is executed, the matching contour segments are identified only for retained image couples; otherwise it is executed for all possible input fragment couples. We have experimented by combining the dynamic programming algorithm with several color quantization methods. We have also evaluated the performance of the matching contour algorithm introduced. The selection of the specific performance metrics is justified by the fact that most of the contour matching approaches, lead to the extraction of "estimate" matching contour segments that overlap with the "correct" matching contour segments.

The matching performance depends on the quality of the overlap, which is estimated by the ratio of unidentified and mis-identified contour pixels, or false negative and false positive rate. The final step is the overall image reassembly. We evaluated the proposed method with/without employing the first step (the discovery of probably adjacent image fragments) and compared it with the manually (human) reassembly.

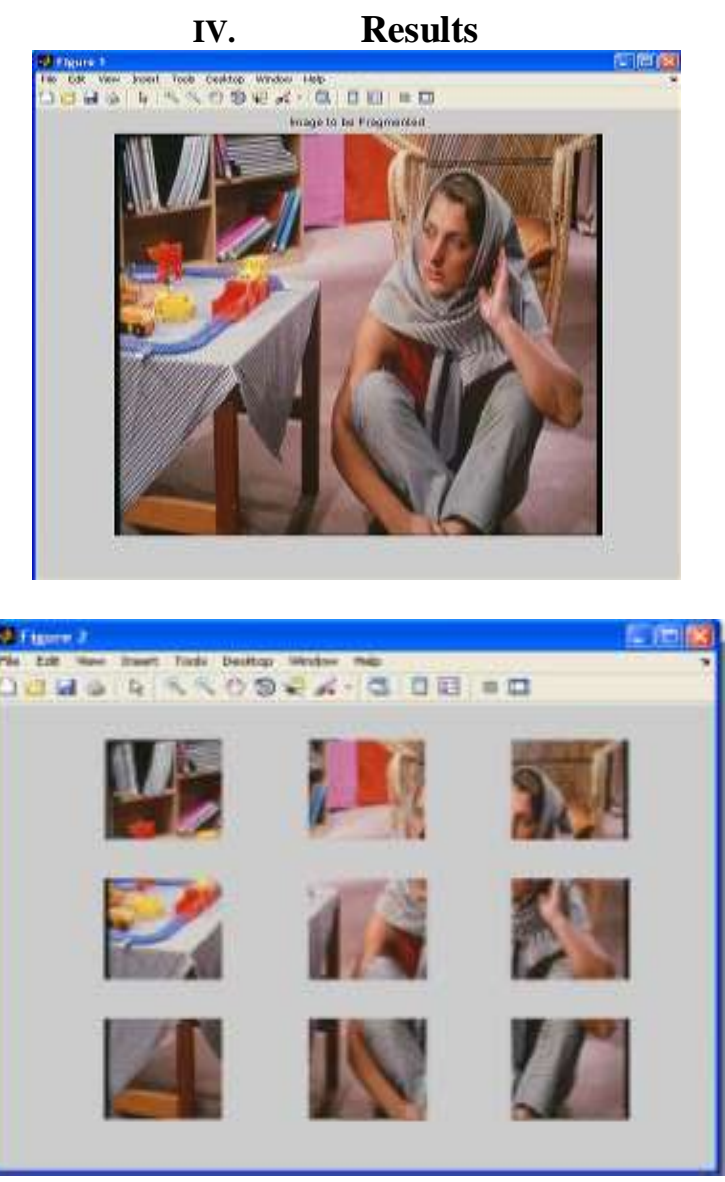



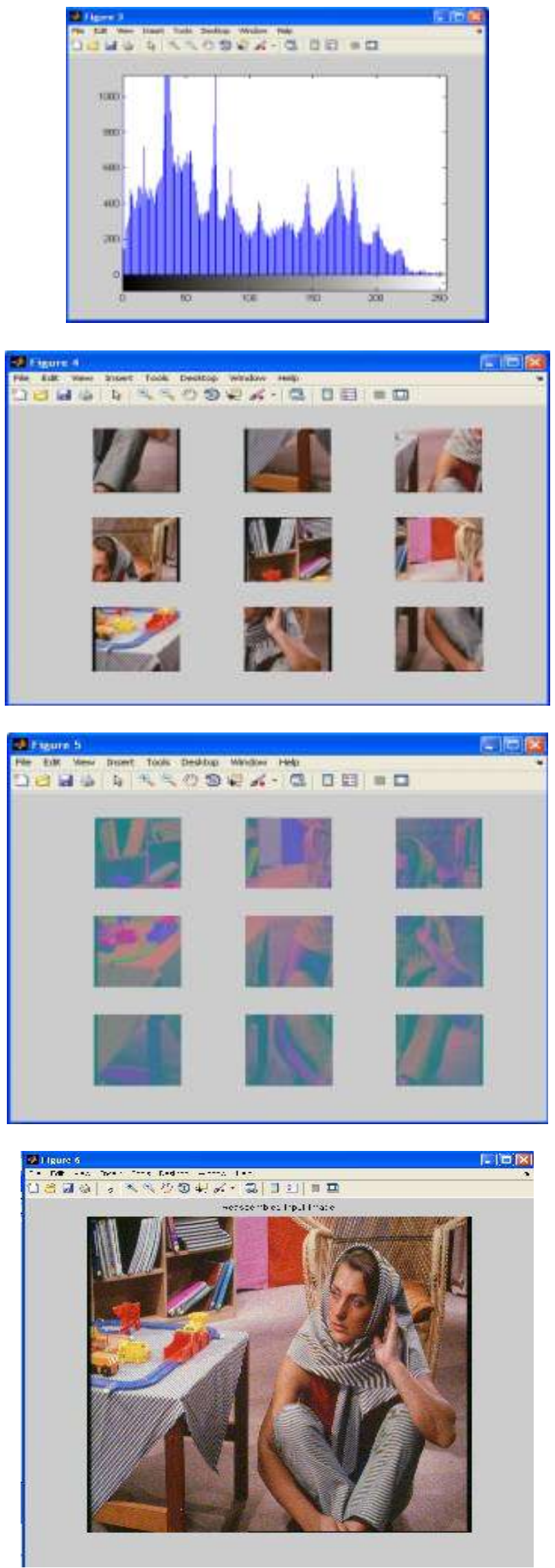

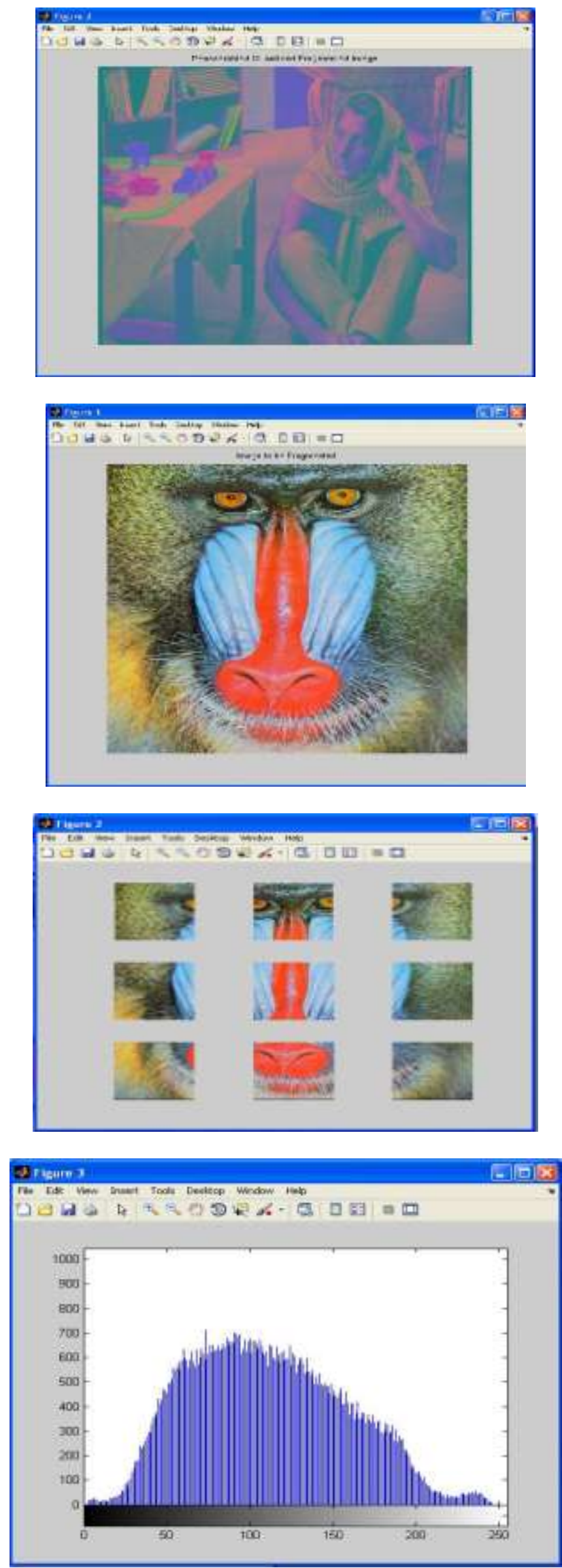


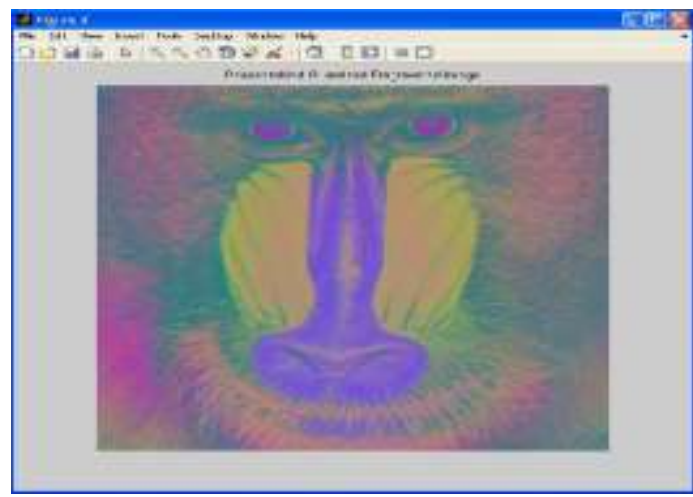

We have averaged the time needed for each person to correctly reassemble each fragmented image. As expected, the overall performance will be higher when the first stage is omitted. However, in this case the computation cost is higher, since both the second and the third step are executed for every couple of input image fragments. In the first case, where the the amount of fragments is somewhat small (10 fragments), the reassembly time efficiency is high in every case (with/without first step, manually reassembly). As the amount of fragments per input image increases, the time efficiency deviations become higher. The choice whether to employ the first step on the reassembly process depends on the computational cost that is associated within the input image fragments. As the amount of image fragments increases, the higher overall performance of the variation that omits the first step gives way to the higher computational cost that is associated with it.

\section{Conclusion}

The overall assembly performance has proven to be particularly satisfactory. The experiments were conducted with 85 paper image prints. We plan to further improve the performance of the proposed method. Improvements can be done in each step of the proposed method. For example, the step of the discovery of spatial adjacent image fragments may be improved by employing not only color but textual or semantic features as well. Another possible extension is to utilize both the color and the shape of the fragments contours in order to perform matching. Finally, the evaluation of the proposed method in a realistic fragmented image database, such as archaeological data, is worth exploring.

\section{References}

[1] A. Kalvin, E. Schonberg, J. Schwartz, and M. Sharir, "Two dimensional model based boundary matching using footprints," Int. J. Robot. Res., vol. 5, no. 4, pp. 38-55, 1986.

[2] D. Goldberg, C. Malon, and M. Bern, "A global approach to automatic solution of jigsaw puzzles," Computat. Geom.: Theory Appl., vol. 28, pp. 165-174, 2004.

[3] W. Kong and B. B. Kimia, "On solving 2D and 3D puzzles using curve matching," in Proc. IEEE Computer Society Conference on Computer Vision and Pattern Recognition, 2001, vol. 2, pp. 583-590.

[4] G. Papaioannou, E. A. Karabassi, and T. Theoharis, "Reconstruction of three-dimensional objects through matching of their parts," IEEE Trans. Pattern Anal. Mach. Intell., vol. 24, no. 1, pp. 114-124, Jan. 2002.

[5] S. Andrews and D. H. Laidlaw, "Toward a framework for assembling broken pottery vessels," in Proc. 18th American Conf. Artificial Intelligence, 2002, pp. 945-946, American Association for Artificial Intelligence (AAAI).

[6] S. Rusinkiewicz and M. Levoy, "Efficient variants of the ICP algorithm," in Proc. 3rd Int. Conf. 3D Digital Imaging and Modeling (3DIM), Jun. 2001, pp. 145-152.

[7] G. C. Sharp, S. W. Lee, and D. K. Wehe, "Icp registration using invariant features," IEEE Trans. Pattern Anal. Mach. Intell., vol. 24, no. 1, pp. 90-102, Jan. 2002.

[8] D. Arthur and S. Vassilvitskii, "Worst-case and smoothed analysis of the icp algorithm, with an application to the kmeans method," in Proc. 47th Annu. IEEE Symp. Foundations of Computer Science (FOCS), 2006, pp. 153-164. 